\title{
Hormonal treatment of vulvar vaginal atrophy (VVA): Are there options to reduce or avoid systemic adverse effects and risks?
}

\author{
Xiangyan Ruan ${ }^{1,2}$ and Alfred O Mueck ${ }^{1,2 *}$ \\ ${ }^{1}$ Department of Gynecological Endocrinology, Beijing Obstetrics and Gynecology Hospital, Capital Medical University, China \\ ${ }^{2}$ University Women's Hospital and Research Center of Women's Health, Department of Women's Health, University of Tuebingen, Germany
}

\begin{abstract}
Vulvar vaginal atrophy (VVA) is very frequent, about half of postmenopausal patients suffer from this disease. Since VVA is mainly caused by estrogen deficiency, the present first-line recommendation in guidelines is estrogen therapy. Because of the fear of systemic absorption, vaginal application is recommended if only VVA should be treated, to avoid known estrogen-dependent risks like venous thromboembolism, stroke and hormone-dependent cancer. A new alternative to estrogens is ospemifene from the class of "selective estrogen receptor modulators" (SERMs) which is thought to act selectively as an agonist on estrogen receptor alpha in the vaginal tissue. The aim of this review is to describe the conventional management using different types and dosages of vaginal estrogens and to compare with the treatment using ospemifene. Thereby the focus is on tolerability and safety issues including the official warnings in the labelings of both treatment strategies. Especially also the new concept to use "ultra-low dose" estriol alone or in fix combination with lactobacilli is highlighted, because this therapy perhaps can avoid systemic estrogenic effects although according present labeling in patients after breast cancer treatment only ospemifine can be used. Thus, this review on both treatment strategies should be important for the clinical practice.
\end{abstract}

\section{Introduction and aim of this review}

Approximately $50 \%$ of postmenopausal women suffer from VVA symptoms [1]. Vulvar and vaginal atrophy (VVA) are mainly caused by the decrease of estrogen production during peri- and postmenopause [2-4]. Thus systemic or vaginal estrogen-based treatments are currently recommended and approved by the U.S. Food and Drug Administration (FDA) and other health authorities like the European Medicines Agency (EMA), and in the different guidelines also are recommended as first-line therapy [2-9]. Systemic administration of estrogen as a part of post-menopausal hormone therapy would ameliorate urogenital atrophy, but after the increased risk of venous thromboembolism, stroke and breast cancer, assessed in the Women's Health Initiative (WHI) trial [10], many women no longer choose such a therapy [11].

To avoid or reduce possible adverse effects and the risks known from systemic administration of estrogens, vaginal estrogen therapy is generally recommended, unless other symptoms of menopause, such as hot flushes, are present [2-9]. However, there remains some fear that vaginal estrogen treatment may be absorbed in the circulation and increase adverse systemic estrogen-dependent effects [12,13]. Despite good evidence is lacking, the labeling of the commercial available vaginally applied estrogens regarding contraindications and warnings is mainly the same like for systemically applied estrogens which is due to the "class-labeling", in general often performed by health authorities. The question is, if those side effects and risks may be dependent or different by the type of estrogens used vaginally and if the risk of systemic action could be reduced with low-dose or with new "ultra-low dose" estrogen products.

Another option to avoid unwanted systemic effects would be products which despite systemic application (like orally) only are acting in the urogenital tissue and inducing the well-know beneficial estrogen effects for women with VVA. By definition "tissue-selective" products are "Selective Estrogen Receptor Modulators" (SERMs), which can act on the estrogen receptors dependent from the tissue as agonist or antagonist. From the commercially available SERMs, ospemifene has been approved for symptoms of VVA due to menopause [14-17]. Although the approvement has the limitation that ospemifene only should be used for women with VVA, who are not candidates for local estrogens, i.e. who are unable to tolerate or unwilling to take vaginal estrogens, this treatment concept may be of great clinical importance, since in contrast to estrogens ospemifene can be used also in patients with breast cancer after treatment including adjuvant endocrine treatment [16,17], in contrast to the labeling of all estrogen products including vaginal applied estrogens.

Ospemifene has been developed especially to avoid adverse effects and risks known from systemic estrogen treatment but with at least the same beneficial efficacy for treatment of VVA. As the corresponding author has been coauthor of two already published papers on the use of ospemifene $[17,18]$, one aim for the present review was to present

${ }^{\star}$ Correspondence to: Alfred O.Mueck, Department of Women's Health University of Tuebingen, Calwer Street 7; D-72076 Tuebingen, Germany, Tel: +49 - 7071-298 4801; Fax: +49 - 7071-29 4801; E-mail: alfred.mueck@med.unituebingen.de; A.O.Mueck@t-online.de

Key words: Vulvar Vaginal Atrophy (VVA), vaginal estrogens, ospemifene, ultralow estriol, lactobacilli, systemic risks

Received: October 09, 2018; Accepted: October 19, 2018; Published: October 22,2018 
a short summary from this research with the special focus on safety and tolerability aspects. The second aim was to compare with the vaginal estrogen treatment as the present standard of VVA therapy including the very special concept using vaginal tablets containing "ultra-low dose" estriol combined with lactobacilli. We just recently have published extensively on the available scientific knowledge of this combination [19], and also for this treatment option the focus in the present review is on tolerability and safety. With these two aims for the present review the question should be answered, if these two treatment strategies for VVA are options to reduce or avoid systemic adverse effects and risks.

\section{Current Management of VVA}

\section{Definition, diagnosis and symptom spectrum of VVA}

The decrease of estrogen production during the menopausal transit induces changes in the vulvar, vaginal and urogenital mucosae which are responsible for clinical symptoms such as dyspareunia, vaginal dryness, itching and burning with potential negative impact on women's sexual function $[6,20,21]$. To describe this medical condition various terms have been used: Vulvovaginal atrophy (VVA), urogenital atrophy, (symptomatic) vaginal atrophy, atrophic vaginitis, colpitis senilis, etc. Recently a new terminology has been suggested by American scientific societies describing the complex of typical urogenital signs and symptoms present in the menopausal women as genitourinary syndrome of menopause (GSM) [9,21]. In the following we describe this complex syndrome as "vulvar and vaginal atrophy" (VVA), since pathophysiology and the clinical symptoms may not exclusively be dependent on menopause, but can be seen also for example after chemotherapy or in women with primary ovary insufficiency etc. $[8,9]$.

For the diagnosis and degree of severity of VVA besides the clinical symptoms objective measurements are used in the routine management as well as for endpoints in clinical studies. Primarily this includes the assessment of the maturation index evaluating the percentage of barabasal and superficial cells and the vaginal $\mathrm{pH}$. Due to the complexity of the clinical syndrome in some studies one symptom or a symptom complex are defined as "most bothersome symptom(s)" (MBS), e.g. vaginal dryness, whereby often scales are used to measure the severity of disease.

The importance of VVA with its most common spectrum of symptoms can for example be derived from the REVIVE (REal Women's VIews of Treatment Options for Menopausal Vaginal ChangEs) Survey aiming to characterize postmenopausal women's experience with VVA, interactions with health-care professionals (HCPs), and available treatment options [22]. This US online survey (the largest US cohort of recent surveys) has been described to be projectable to the overall US population. 3,046 postmenopausal women with VVA symptoms responded to the structured questions. The most common VVA symptoms were dryness (55\%), dyspareunia (44\%), and irritation (37\%). VVA symptoms affected enjoyment of sex in $59 \%$ of participants. Surprisingly, only few women attributed symptoms to menopause $(24 \%)$ or hormonal changes (12\%). Only $56 \%$ had ever discussed VVA symptoms with an HCP, and from these women $62 \%$ used over-the-counter (OTC) products. Significant barriers to treatment include lack of knowledge about VVA, reluctance to discuss symptoms with HCPs, safety concerns, inconvenience, and inadequate symptom relief from available treatments.

\section{Conventional treatment options for VVA}

Regarding non-hormonal treatments of VVA-related symptoms, OTC-available vaginal moisturizers and lubricants are commonly in use. Moisturizers are defined as preparations which can replace the normal vaginal secretions, whereas lubricants are specifically designed to reduce friction during sexual intercourse [23]. Vaginal moisturizers applied on regular basis in higher dosages may have an efficacy comparable to vaginal estrogen application for relieving symptoms of VVA such as itching,irritation and dyspareunia, and should be offered to women wishing to avoid the use of estrogen or if hormonal treatment is contraindicated [24,25]. Vaginal moisturizers and lubricants as well as regular sexual activity may be helpful for women in improving their quality of life. Nonetheless, it has to be emphasized that in the studies the products had been tested mostly only for a duration of three months, and data concerning long-term safety and efficacy are missing. In addition, it is very difficult to rule out the placebo effect. In general, non-hormonal substances including phytoestrogens and special "traditional Chinese medicine" could provide temporary relief of symptoms but have only limited efficacy in the treatment of VVA [9,26-28].

According the role of estrogen deficiency causing VVA as first-line treatment estrogens are recommended [2-9]. Estrogen applications acting systemically (in the following "systemic estrogens") like oral, transdermal, subdermal and intramuscular applications can be effective for the treatment of moderate, even severe symptoms of VVA. The mechanisms of estrogens in treating and reversing the physiological changes associated with VVA may include promoting vaginal cell growth and cellular maturation, enhancing vaginal blood flow, fostering lactobacilli recolonization, decreasing vaginal $\mathrm{pH}$, and increasing vaginal epithelial thickness and elasticity $[20,28,29]$.

Estrogens administered vaginally are absorbed in a dose-dependent manner and bypass hepatic metabolism [30,31]. Vaginal estrogens are more effective in relieving urogenital symptoms than oral preparations because high local estrogen concentrations and absence of hepatic metabolism $[32,33]$. The use of local vaginal estrogens is the option of choice - it provides an effective and safe treatment that dramatically improves vaginal symptoms and restores woman's sexual and general quality of life [20]. In severe cases, a combination of systemic and local hormone therapy may be required initially, as systemic estrogen therapy alone relieves vaginal atrophy symptoms in only about $75 \%$ of women [34].

However, although vaginal estrogen therapy is intended to have only local effects on the urogenital organs, the fear is that estrogens are absorbed into the bloodstream to an extent that systemic effects can occur $[12,13]$. There may be especially an increased risk of endometrial hyperplasia, perhaps partly due to estrogen penetrating form the vagina into the uterine cavity, besides estrogen absorption into the blood circulation $[3,12,28]$. This so called "uterine first-pass effect" is thought also to be responsible for a higher bioavailability of vaginal applied estrogens compared to the oral application as this also has been discussed for the higher bioavailability and stronger endometrial action of progesterone applied vaginally compared to oral medication [35].

Different types and preparations of vaginal applied estrogens have been used for many years including estradiol-containing tablets and rings, estriol pessaries, creams and ovules, promestriene and conjugated estrogens, with different availibility in the various countries. A Cochrane review [28] identified 19 trials with 4,162 women from a total of 37 trials comparing the efficacy of estrogenic preparations for 
the treatment of VVA. The treatment with vaginal estrogens in general was significantly superior compared to placebo and non-hormonal alternatives. Regarding safety, with vaginal conjugated equine estrogens (to our knowledge not available in Europe) or using the estradiol ring, systemic adverse effects like breast pain, uterine irregular bleedings and the risk of endometrial hyperplasia could not be excluded.

\section{Vaginal Estradiol for treatment of VVA}

Increased risks due to systemic action have also been discussed for the use of vaginal estradiol tablets frequently used especially in Scandinavian countries but also in USA and Canada. $25 \mu \mathrm{g}$ tablets were first introduced in 1988, and in 2009 the FDA approved a $10 \mu \mathrm{g}$ formulation, both vaginal tablets should be inserted twice a week.

Both formulations have been compared within a randomized, double-blind, multicenter US study during treatment of 230 postmenopausal for three months, and VVS was relieved significantly with both dosages [36].Within a follow up multicenter study performed in USA and Canada in 309 postmenopausal women comparing 10 $\mu \mathrm{g}$ E2 vs placebo [37] the estrogen tablets significantly improved during treatment of one year vaginal cytology and $\mathrm{pH}$ and a score defined for the most bothersome urogenital symptoms. In this study one endometrial adenocarcinoma stage II, grade 2 was found in the estradiol group. However, a causal relationship remained unclear because for treatment of hot flushes previously systemic unopposed estrogen has been used which certainly can induce endometrial cancer, and it was unclear if there already has been a preexisting cancer which was not detected during the baseline biopsy. Within an European study including 336 postmenopausal women [38] using $10 \mu \mathrm{g}$ E2 for one year and assessing endometrial histology pre- and posttreatment no endometrial hyperplasia or cancer were detected which should be reassuring regarding endometrial safety. However, regarding treatment in patients with breast cancer vaginal estradiol formulations should not been used during endocrine adjuvant treatment with aromatase inhibitors because during the first weeks of treatment relevant systemic absorption could occur [39]: Using the dose of $25 \mu \mathrm{g}$ vaginal E2 in a study with seven postmenopausal women resulted in an increase of E2-levels from base-line levels $\leq 5 \mathrm{pmol} / \mathrm{l}$ up to a mean of $72 \mathrm{pmol} / \mathrm{l}$ [40]. Within another study in breast cancer survivers using only 12.5 $\mu \mathrm{g}$ vaginal estradiol lower levels have been observed [41], but also these authors warned for therapy using vaginal estradiol in patients after breast cancer.

Those warnings because of possible systemic adverse effects and risks have been recently supported by the results of quite another study investigating not possible systemic risks but possible benefits using vaginal estradiol [42]. Analyzing in total 195,756 postmenopausal women within a nationwide cohort in Finland exposed during 19942009 exclusively to vaginal applied estradiol, the aim was to investigate if this topical treatment could reduce the mortality risk for cardiovascular diseases as this has been shown within studies using oral or transdermal estrogens. In this Finnish register study $98,3 \%$ of the study population used $25 \mu \mathrm{g}$ E2 (vaginal tablets twice a week) and 1,7\% used a vaginal ring releasing E2 $7.5 \mu \mathrm{g} /$ day, which still was a quite large patient sample $(\mathrm{n}=3,286)$. The follow-up data gathered 1.4 million women-years, and the mortality risk due to coronary heart disease (CHD) $(n=9,656)$ or stroke $(n=4,294)$ was assessed. Surprisingly a reduction for both CHD and stroke mortality was detected in all age groups with the highest risk reduction in women aged 50-59 years (Standardized Mortality Ratio SMR $0.43 ; 0.19-0.88$ and SMR $0.21 ; 0.06-0.58$, respectively). Despite the limitations and reasons for caution which the authors in detail have listed in their publication this study suggest that vaginal estradiol may lead to clinical relevant systemic effects, in terms of beneficial but also adverse action.

It should be mentioned that with the vaginal ring used in the Finnish study releasing $7.5 \mu \mathrm{g}$ estradiol/day beneficial systemic effects already have been reported in earlier studies - during 6-monthly treatment an increase of forearm bone density and improvement of the serum lipid profile has been observed $[43,44]$. From those results we like to conclude that vaginal estradiol, independent of the topical applied product form as tablets or ring, may in general not be an option to avoid adverse systemic effects, and it should be not used in patients known to be at increased risk like venous thromboembolism and hormone-dependent cancer. Especially due to the absorption into blood circulation, vaginal estradiol is not recommended for women with a history or during treatment of breast cancer, particularly if they are using aromatase inhibitors [45]. In the meantime the $25 \mu \mathrm{g}$ formulation of vaginal estradiol is no longer available in Europe [17].

Recently within the large prospective Women's Health Initiative (WHI) Observational Study 45,663 postmenopausal women using vaginal estrogens were recruited at 40 US clinical centers, aged 50 to 79 years at baseline, with median follow-up of 7.2 years [46]. Among women with an intact uterus, the risks of stroke, invasive breast cancer, colorectal cancer, endometrial cancer, and pulmonary embolism/ deep vein thrombosis were not significantly different between vaginal estrogen users and nonusers, whereas the risks of CHD, fracture, all-cause mortality and a special defined "global index event" (GIE) were lower in users than in nonusers (GIE adjusted hazard ratio 0.68 , 95\% confidence interval $0.55-0.86$ ). These results should provide reassurance about the safety of treatment using vaginal estrogens, but comparable to the above described Finnish study [42] also must clearly lead to the conclusion, that vaginal estrogens also can act systemically. Unfortunately, the specific type and formulation of vaginal estrogen (cream, ring, or tablet) or dose was not collected, and because various limitations described in the publication [46] (e.g. only data collection using selfassessments) caution is suggested regarding definitive conclusions about safety of vaginal estrogen treatment.

\section{Vaginal Estriol for treatment of VVA}

Estriol (E3) preparations (pessaries, creams and ovules) have been used already since many years. Most applications provide a dosage of 0.5 or $1 \mathrm{mg}$, whereby treatment is mostly started with daily application followed later twice weekly. E3 has been defined as a short-acting estrogen, since it has the shortest receptor occupancy and lowest receptor affinity of all natural estrogens. The mechanism of action in vagina and uterus are similar like with E3 and E2 but with the difference of a 10-fold lower affinity on the estrogen receptor for E3 versus E2 [32,47,48]. Whereas E2 and estrone (E1) can be reversibly metabolized into each other, E3 is a final metabolite of the estrogen synthesis $[47,49,50]$. These pharmacological properties contribute that possible systemic side effects are lower compared with other estrogens, in addition to only a very low risk of relevant systemically absorption which has led to the suggestion that estriol, but not other local applied estrogens could be used after breast cancer diagnosis [51].

In addition it was suggested that once-daily treatment with intravaginal E3 $(0.5 \mathrm{mg})$ in postmenopausal women should be also without increased risk of endometrial hyperplasia [52]. Thus addition of a progestogen like this is mandatory using oral or transdermal estrogen in women with uterus, is not recommended, and monitoring of endometrial thickness using vaginal estrogens in the routine 
management of VVA is not needed [2,7-9,53]. However, because the effect on both breast and endometrial tissue are known to be dependent on hormone type, dose and frequency of administration, this is recommended during continuous vaginal use of high doses of 'potent' estrogens like estradiol.

Compared with other types of estrogens, with the use of estriol there have been already for long time the lowest safety concerns [32]. However, it seems that the conventional therapy used for decades can be further optimized by achieving comparable efficacy with further lowering any possible risk of systemic adverse effects using the "E3ultra-low dose" therapy for treatment of VVA, with the consens that this therapy may be even possible for high-risk patients like after diagnosis of breast cancer [26,54-60]. This concept has got to be included in current recommendations especially for risk patients latest with the results of a carefully performed multicenter, randomized, placebo-controlled, double-blind study evaluating lower dosages [58]: 436 postmenopausal women with vaginal atrophy were treated with pessaries containing either $0.2 \mathrm{mg}$ estriol $(\mathrm{n}=142)$ or $0.03 \mathrm{mg}$ estriol $(\mathrm{n}=147)$ or a matching placebo $(\mathrm{n}=147)$ for 12 weeks. Both doses were significantly more effective than placebo using for the diagnosis of VVA objective and subjective parameters. Using a visual analog scale (VAS) the "most bothersome symptoms (MBS)" (vaginal dryness, pain, redness, itching, dyspareunia) were reduced to about $60 \%$. Despite it was demonstrated, that with this "ultra-low dose" treatment using E3-0.03 mg pessaries still E3-levels can be assessed, no systemic side effects should be expected, since already $12 \mathrm{~h}$ after administration the levels dropped from $42 \mathrm{pg} / \mathrm{ml}$ to lower than $5 \mathrm{pg} / \mathrm{ml}$, which has been the lowest limit of assay sensitivity. Repeated administration during 21 days did not result of an accumulation of estriol reaching a maximum level of only $11.9 \mathrm{pg} / \mathrm{ml}$ [55].

\section{SERMs for treatment of VVA}

A class of compounds with the potential to treat VVA are the selective estrogen receptor modulators (SERMs). These compounds mediate their effects primarily through the estrogen receptor- $\alpha(E R \alpha)$ and can have selective estrogen agonist or antagonist effects depending on the target tissue and endpoint. Tamoxifen and raloxifene are FDA- and EMA-approved SERMs; however, neither is appropriate for systemic treatment of vaginal atrophy. Raloxifene does not have any significant positive effect on the vagina, neither assessing clinical endpoints nor with respect on objective parameters like vaginal $\mathrm{pH}$ or maturation index [61-63]. Tamoxifen has both estrogen agonist and antagonist effects on the vaginal epithelium [64]. Although longterm tamoxifen treatment may have estrogenic effects on the vaginal epithelium, adverse vaginal effects during treatment with tamoxifen, including dyspareunia and vaginal dryness, have been reported. Especially due to other possible long-term adverse effects (e.g. risk of endometrial cancer) the benefit-risk ratio would raise concern if tamoxifen would be used only for treatment of VVA.

Ospemifene has been primarily developed as a SERM which despite oral application should selectively act as estrogen agonist in the vaginal tissue [17,65-67]. Using this SERM for treatment of VVA as an alternative to estrogen treatment was thought to avoid typical risks known from estrogens like venous thromboembolism and stroke or hormone dependent cancer like endometrial and breast cancer.

\section{Ospemifene, oral SERM for treatment of VVA}

\section{Ospemifene - Safety suggestions from a Phase III Study}

The question if the use of ospemifene may avoid adverse systemic effects will be discussed in context with a study, which in details already has been published as an original paper together with the corresponding author of the present review [18]. The primary aim of this study was not to assess the safety, but the efficacy of ospemifene in the treatment of VVA in postmenopausal women [18]. The following discussion will focus on the safety and tolerability evaluations which could be suggested from this study, although this was not a primary endpoint.

The design of the trial was a 12 week, multicenter, randomized, double-blind, parallel-group phase III study, performed in the USA. The detailed study design including screening, recruitement, randomization, describing ITT- and PP-population and methods for evaluation of primary and secondary endpoints, has been described elsewhere $[18,67]$. Primary efficacy endpoints of the study were the change from baseline to week 12 in (1) percentage of parabasal cells in the maturation index, (2) percentage of superficial cells, (3) vaginal $\mathrm{pH}$, and (4) severity of vaginal dryness. 314 women were randomised to once-daily ospemifene $60 \mathrm{mg} /$ day $(n=160)$ or placebo $(n=154)$. Improvements in each of the four primary endpoints with ospemifene were statistically significant compared to placebo.

The majority of treatment-emergent adverse events were considered mild to moderate in severity. Adverse events (AEs) have been rare and the majority were rated as mild or moderate. However, they have been more frequent during ospemifene compared to placebo ( 65 vs $50,6 \%$ of patients) although most were classified as not related or unlikely to be related to the study drug (66.5\%). The most frequently reported AEs in the ospemifene group were urinary tract infection (12.5\% of patients), hot flushes (7.5\%) and nasopharyngitis (5.6\%). Hot flushes during treatment with SERMs in general relatively often has been described suggesting that the "selective estrogen receptor modulation" may not work "selective" in all patients since in the brain the ideal SERM should act as an ER agonist reducing or avoiding hot flushes [65]. However, only three patients discontinued the study due to worsening of hot flushes during treatment with ospemifene.

In this context one of the two observed "serious AEs" was a deep vein thrombosis (DVT) and was considered as probably treatment related. From various studies using hormone therapy we know that hormones in oral application can increase the risk of DVT within short treatment. However, this study cannot give any suggestions about relative or absolute DVT risk because of the small sample size.

In contrast to DVT the development of endometrial cancer and breast cancer would need longer treatment. However, it never can be excluded that a patient with preexisting cancer cells could get "clinical cancer" during short-term treatment if the drug could induce proliferation. In this study no breast cancer was observed, and also no cases of endometrial hyperplasia, endometrial polyps, or carcinoma were found in the endometrial biopsy samples. Vaginal bleeding was reported in two patients in each treatment group (ospemifene and placebo), i.e. also derived from bleeding patterns there should be no concern.

A slight increase in the mean endometrial thickness in women receiving ospemifene could be seen. More important than mean values of endometrial thickness (often only presented in studies!) is the question about the numbers of patients who present proliferation beyond a certain threshold, e.g. $\geq=5 \mathrm{~mm}$. In this study, within three months, this was observed only for three patients $(3 / 51 ; 5.9 \%)$ in the ospemifene group and one patient $(1 / 58 ; 1.7 \%)$ in the placebo group. Two patients $(2 / 51 ; 3.9 \%)$ in the ospemifene group had an endometrial thickness $\geq 8 \mathrm{~mm}$ at week 12 . 


\section{Ospemifene - systemic adverse effects should not be excluded}

From the above results of one special trial, described as an example within the extensive research during the development of ospemifene, we can conclude that during treatment with ospemifene systemic adverse events, including endometrium-proliferating effects, are very rare but should not be excluded. However, in general in the studies ospemifene was assessed as to be safe and well tolerated $[17,67]$. Based especially on randomized controlled trials, oral ospemifene $60 \mathrm{mg}$ showed an overall positive risk-benefit profile in postmenopausal women with VVA and was from the FDA in 2013 approved for the treatment of "moderate to severe dyspareunia, a symptom of vulvar and vaginal atrophy, due to menopause" (US labeling) [14,15] or "indicated for the treatment of moderate to severe symptomatic vulvar and vaginal atrophy (VVA) in post-menopausal women who are not candidates for local vaginal oestrogen therapy" (European labeling) [16,17], respectively. Up today the efficacy and safety of ospemifene were established in about 30 clinical trials, with about 2,500 patients exposed to ospemifene [17].

In terms of possible systemic adverse effects hot flushes also have occurred more frequently in other studies $[67,68]$. So certainly, ospemifene may increase the incidence of hot flushes and is not effective in reducing hot flushes associated with estrogen deficiency. A 12-month study of endometrial safety with ospemifene $(n=426)$, confirmed minimal stimulation of the endometrium, but with only one case of simple hyperplasia, representing a rate of $0.3 \%$, which is lower than the known spontaneous rate of 1\% [69]. So derived from the endometrial biopsies this study would support the tissue selectivity of ospemifene, but taken all data together, stimulation of endometrial proliferation, a key consideration for chronic use of all SERMs [70], should not be excluded.

\section{Ospemifene - Labeling, Warnings, comparing with estrogens}

In the FDA-labeling a"black box-warning" in terms of the risk of endometrial cancer has been included [14,15]: "... In the endometrium, ospemifene has estrogen agonistic effects. There is an increased risk of endometrial cancer in a woman with a uterus who uses unopposed estrogens. Adding a progestin to estrogen therapy reduces the risk of endometrial hyperplasia, which may be a precursor to endometrial cancer. ". For the reader this direct combination of warnings in terms of ospemifene use and estrogen use would suggest similar risks, which, however, to our knowledge have not been demonstrated in adequate clinical studies. Also the use of progestins added to ospemifene has not been evaluated. Nevertheless, clinical surveillance of all women using ospemifene is warranted. Besides the routine diagnostic in cases of suspected endometrial hyperplasia or cancer, which includes endometrial histology, patients always should be asked about bleedings, which are extremely rare during therapy with ospemifene. If bleeding or spotting occurs, the therapy should be discontinued and the reason for bleeding should be investigated.

Regarding the risk of breast cancer - this has not been included in the primary FDA-black box warning although "known or suspected estrogen-dependent neoplasia"are listed as contraindications [15]. According to the labeling of the European product [16], ospemifene ".... has not been formally studied in women with a prior history of breast cancer. No data are available on its concomitant use with medicinal products used in the treatment of early or advanced breast cancer. Therefore it should be used for the treatment of VVA only after the treatment of breast cancer, including adjuvant therapy, has been completed".
In terms of cardiovascular diseases there is a warning in the "FDA black box [15]: "In the clinical trials (duration of treatment up to 15 months) the incidence rates of thromboembolic and hemorrhagic stroke were 0.72 and 1.45 per thousand women, respectively, in the ospemifene treatment group and 1.04 and 0 in placebo. The incidence of DVT was 1.45 per thousand women in the ospemifene treatment group and 1.04 per thousand women in placebo". The warning is, like the warning for the risk of endometrial cancer, directly combined with the warning in terms of the risk if estrogens are used, although we are not aware that the risks have been head-to-head evaluated or assessed in comparable trials: "There is a reported increased risk of stroke and deep vein thrombosis in postmenopausal women (50 to 79 years of age) who received daily oral conjugated estrogens (CE) [0.625 $\mathrm{mg}$-alone therapy over 7.1 years as part of the Women's Health Initiative (WHI)."

Perhaps the warning on cerebrovascular disease and deep vein thrombosis was included in the labeling because these risks have been seen with other SERMs, so these risks should not be excluded if ospemifene is prescribed. However, future research is needed comparing with the present recommended first-line treatment using vaginal estrogen therapy.

\section{Comparison of ospemifene versus conventional vaginal estrogens}

Until today to our knowledge direct comparisons between oral ospemifene and vaginal estrogens have not been performed. In the absence of direct (head-to-head) comparisons in randomized clinical trials and also because it was requested of EU regulatory authorities, an indirect historical comparison was performed of oral ospemifene 60 mg vs. local estrogens that are currently available for VVA treatment in Europe. This extensive comparison has been published with the participation of the corresponding author of the present review [17]. Thus here will be given only a short summarizing extract of this comparison, particularly in terms of safety issues.

Of the 88 clinical studies on local estrogens according to our selection criteria [17] only 10 could be used for the safety analysis including estriol 0.5 or/and $1 \mathrm{mg}$ [71,72], vaginal ring releasing E2 7.5 $\mu \mathrm{g} /$ day [73-76], and vaginal E2 $10 \mu \mathrm{g}$ [36-38,77] (vaginal E2 $25 \mu \mathrm{g}$ no longer available in Europe!), whereby five studies had not comparator arm [38,71-73,76], one has an untreated control [74], one higher dosage estradiol [75] and three were placebo-controlled [36,37,77]. Ospemifene $60 \mathrm{mg}$ long-term safety data were obtained from the pivotal 52-week, double-blind, placebo-controlled trial [69] and the blinded 40-week extension from one of the pivotal 12-week, doubleblind, placebo-controlled trials [78].

Regarding all efficacy comparisons, short and long-term with different endpoints, this has been in detail described [17]. In summary there were no clinical relevant differences with a tendency of stronger efficacy using ospemifene. Regarding safety in the included studies using vaginal estrogens, three $[73,75,76]$ reported vaginal bleeding, which was not seen in the studies with ospemifene. Mean endometrial thickness was reported in three estrogen studies [73,75,76], all remained unchanged over one year of treatment whereas with ospemifene a small increase over 52 weeks was seen [78]. Endometrial biopsies at 12 months were reported with vaginal E2 $10 \mu \mathrm{g}$ with one event of carcinoma and one complex hyperpasia [77]. No endometrial carcinoma with ospemifene was observed. There also were no cases of breast cancer in the ospemifene studies, but also none reported in the estrogen studies. However, it has to be considered that according the labeling history of breast cancer is a contraindication for all 
estrogens including local estrogens whereas ospemifene can be used after (adjuvant) treatment has been completed. No cases of venous thromboembolism in the studies with local estrogens have been reported, in contrast to ospemifene, where the incidence was calculated to be $3.65 / 1000$ women-years. The same incidence, however, was seen in the placebo population.

As expected with the use of a SERM, there have been more hot flushes using ospemifene compared with placebo. Vaginal candidiasis, a known side effects of topical estrogens, was more often seen with both, ospemifene $(7,7 \%)$ and vaginal E2 (8,3\%) compared to placebo $(1,6 \%$ and $2,9 \%$, resp..). The incidence of headache compared with placebo was not increased with ospemifene, whereas it was with vaginal E2, which confirms relevant systemic absorption. As known with other SERMs, the incidence of muscle spasm was higher with ospemifene than with placebo and was not reported with vaginal estradiol. The majority of muscle spasms observed in the ospemifene phase $2 / 3$ study program were reported as mild or moderate leg cramps. Nevertheless, if observed, this should imply careful examinations to exclude venous thromboembolism which can present with similar symptoms.

The conclusion of this historical indirect comparison was that in the treatment of VVA ospemifene in the safety and tolerability profile is comparable with local vaginal estrogens [17]. It has to be stressed, that in this systematic indirect (historical) comparison versus ospemifene, the new concept of local "ultra-low dose Estriol" in treatment of VVA was not included.

\section{Vaginal "ultra-low dose" estriol combined with lactobacilli for treatment of VVA}

The treatment of VVA using vaginal estriol could be further optimized using a vaginal tablet containing the combination of 0.03 mg E3 combined with lactobacilli. This estrogen dosis is about 15 30 fold lower compared with the "conventional" management which includes dosages of $0.5 \mathrm{mg}$ or $1 \mathrm{mg}$ E3, respectively! Unfortunately until now it is available only in a few countries but it has got already large awareness. We just recently have published an extensive review covering the current relevant knowledge of the pharmacology and clinic of this combination (0.03-E3/L) [19]. We are summarizing here particularly safety and tolerability aspects.

\section{Dual beneficial vaginal effects}

Studies using vaginal $0.03 \mathrm{mg}$ E3-only (0.03-E3) have shown an efficacy comparable with the conventional dosages [58]. Using such a low dose with minimal E3 levels and no cumulation during repeated administration should avoid any relevant effects due to systemic actions. Addition of lactobacilli will further increase particularly the efficacy. The vaginal tablets 0.03 -E3/L contain Lactobacillus acidophilus KS400 strain which is of human origin and with beneficial properties for vaginal use [79]: It produces lactic acid, hydrogen peroxide, adheres well to epithelial cells, and thus inhibits the adherence and growth of relevant vaginal pathogens [80]. Beneficial vaginal lactobacilli also have positive immune modulating properties [81]. Additional defence mechanisms of probiotics, like production of bacteriocins and biosurfactants, co-aggregation and lactobacilli biofilm formation, have been also described [82].

Vaginal tablets E3-03/L can be used due to its dual mechanism (estrogenic effects plus beneficial properties of lactobacilli) for diseases where the establishment and maintenance of a healthy vaginal ecosystem is important: (1) restoration of the vaginal flora; (2) vaginal discharge of unknown origin, where anti-infective therapy is not necessary; and (3) VVA, also as co-medication to systemic hormone therapy. Usually, a dose of 1 vaginal tablet per day over 12 days is recommended as initial treatment; afterwards this can be followed by a maintenance phase of 1 vaginal tablet 2-3 times weekly.

\section{Pharmacokinetics and safety}

For safety issues the question about possible systemic resorption is important: In addition to the above described pharmacokinetic study using 0.03 -E3 only [55] also with $0.03-\mathrm{E} 3 / \mathrm{L}$ was demonstrated that after repeated administration there is no cumulation [83]. A study in postmenopausal breast cancer survivors treated with aromatase inhibitors and suffering from severe VVA demonstrated that a single vaginal application of $0.03-\mathrm{E} 3 / \mathrm{L}$ results only in a small and transient increase of serum E3 (up to $44 \mathrm{pg} / \mathrm{mL}$ ) in $50 \%$ of the women at the beginning of the treatment [84]. After repeated daily vaginal application of the combination the absorption of E3 was not detectable anymore. The observation that serum levels of other sex hormones and their binding proteins were not influenced can lead to the conclusion that there are no systemic actions at all, since especially the hepatic production of binding proteins is very sensitive to any estrogen effect. The decrease in E3-level after repeated vaginal application presumably is caused because of increased local metabolism within epithelial maturation, improved during the initial therapy: E3 absorption via a well-proliferated vaginal epithelium is markedly lower than that via an atrophic one [54,84-86]. Derived from those pharmacokinetic studies it was suggested, that the use of $0.03-\mathrm{E} 3 / \mathrm{L}$ is safe without risk of endometrial or other systemic effects and may be considered for patients with a history of breast cancer and treated with aromatase inhibitors $[84,87]$.

The evaluation of the safety of $0.03-\mathrm{E} 3 / \mathrm{L}$ with respect to clinical endpoints has been evaluated in more than 4,000 women in various clinical studies. In total only $1.7 \%$ adverse drug reactions were observed. $80 \%$ of the side effects were local reactions [79]. There was no evidence of typical side effects risks known from systemic estrogen treatment (thromboembolic events, endometrial stimulation, feeling of tension in the breasts, migraine, nausea, increase in blood pressure, bleeding from the uterus, etc.).

\section{Conclusion}

\section{Which hormonal treatment of VVA to reduce systemic risks?}

Using ospemifene, the labeling of the commercial product includes warnings which seem to compare with the risks observed with estrogens (endometrial cancer, venous thrombosis and stroke), perhaps because systemic effects of ospemifene cannot be excluded and because these risks have been seen with other SERMs. Although similar risks should not be excluded using ospemifene, further research is necessary to quantify possible risks. According to present data, estrogenic adverse effects and risks are certainly very rare. It may well be that in future the product profile of this special drug may be changed, because studies showing positive effects on bone markers, suggesting that ospemifene also acts as an agonist on estrogen receptors in bone. On the other side this clearly points to the fact that ospemifene is not only acting selectively in vaginal tissue but can elicit systemic actions. According to the present labeling ospemifene is an alternative to vaginal estrogen for treatment of VVA whereby it should be used only in women who are unable or unwilling to take vaginal estrogens. However, in contrast to vaginal estrogens it can be used in patients after treatment of breast cancer and there is no limitation according to the duration of treatment. 
Regarding treatment of VVA using vaginal estrogens, in risk patients no estradiol should be used, although with the lower dosage now used in practice ( $10 \mu \mathrm{g}$ in contrast to $25 \mu \mathrm{g}$ ) adverse systemic estrogenic effects seem to be rare. Caution is warranted also in terms of the use of estriol in risk patients in the conventional dosages ( 0.5 or $1 \mu \mathrm{g})$ although with the "twice weekly" concept estrogenic risks also are low. However, the now in some countries available new ultra-low dose formulations containing $0.03 \mu \mathrm{g}$ estriol may be an option also in risk patients, whereby the special concept to combine this ultra-low estrogen with lactobacilli can further improve the efficacy. Considering recent scientific evidence and current treatment guidelines, $0.03 \mathrm{mg}$ E3 formulations or the $0.03 \mathrm{mg}$ E3/lactobacilli combination could be considered as one of the best options for the treatment of VVA in ageing menopausal women.

However, we like to mention, that there is perhaps no concept which may not be able for further improvement: There is that very new discussion about vaginal administration of DHEA which according to a new science of "intracrinology" is thought to replace exclusively missing cell-specific intracellular estrogens and androgens which could avoid systemic estrogenic risks. This new approach is a special strategy concept which is beyond the aim of the present review.

\section{Declaration of interest}

There was no funding for this article. Both authors have received funding for research, advisory, and educational activities from companies which manufacture and/or promote hormone therapy (including local estrogen therapy) or SERMs. Both serve on the board of various societies and journals covering the field of menopause and/ or gynecological endocrinology. The corresponding author presently is the President of the German Menopause Society and the first author President of the Chinese Society of Gynecological Endocrinology affiliated to the International Society of Gynecological Endocrinology. Both authors have founded the first official menopause clinic in China.

\section{Acknowledgement}

Since issues of this review includes to discuss papers which already have been published with the participation of the authors of the present review [17-19], we like to thank all coauthors of these three publications which has given us the possibility to present in the present review our view focused on the question, if the treatment options described in those publications could avoid or at least minimize systemic adverse effects and risks. The literature on ultra-low dose estriol combined with lactobacilli has been searched and summarized, besides with the coauthors of the already published paper [19], also with the help of Medinova AG, Switzerland and Pierre Fabre, Germany.

\section{References}

1. Parish SJ, Nappi RE, Krychman ML, Kellogg-Spadt S, Simon JA, et al. (2013) Impact of vulvovaginal health on postmenopausal women: a review of surveys on symptoms of vulvovaginal atrophy. Int $J$ Womens Health 5: 437-447. [Crossref]

2. North American Menopause Society (2013) Management of symptomatic vulvovaginal atrophy: 2013 position statement of The North American Menopause Society. Menopause 20:888-902.

3. Rahn DD, Carberry C, Sanses TV (2014) Society of GynecologicSurgeons Systematic Review Group. Vaginal estrogen for genitourinary syndrome of menopause: a systematic review. Obstet Gynecol 124: 1147-1156

4. Palacios S, Castelo-Branco C, Currie H, Mijatovic V, Nappi RE, et al. (2015) Update on management of genitourinary syndrome of menopause: A practical guide. Maturitas 82: 308-313. [Crossref]

5. Santen RJ, Allred DC, Ardoin SP (2010) Postmenopausal hormone therapy: an Endocrine Society scientific statement. J Clin Endocrinol Metab 95: s1-s66.
6. Tan O, Bradshaw K, Carr BR (2012) Management of vulvovaginal atrophy-related sexual dysfunction in postmenopausal women: an up-to-date review. Menopause 19: $109-117$

7. Neves-E-Castro M, Birkhauser M, Samsioe G, Lambrinoudaki I, Palacios S, et al (2015) EMAS position statement: The ten point guide to the integral management of menopausal health. Maturitas 81: 88-92. [Crossref]

8. Lumsden MA, Davies M, Sarri G (2016) Guideline Development Group for Menopause D, Management. Diagnosis and Management of Menopause: The National Institute of Health and Care Excellence (NICE) Guideline. JAMA Intern Med 176: 1205-1206

9. Baber RJ, Panay N, Fenton A, IMS Writing Group (2016) 2016 IMS Recommendations on women's midlife health and menopause hormone therapy. Climacteric 19: 109-150. [Crossref]

10. Rossouw JE, Anderson GL, Prentice RL (2002) Risks and benefits of estrogen plus progestin in healthy postmenopausal women: principal results From the Women's Health Initiative randomized controlled trial. JAMA 288: 321-333

11. Ameye L, Antoine C, Paesmans M, de Azambuja E, Rozenberg S (2014) Menopausa hormone therapy use in 17 European countries during the last decade. Maturitas 79 : 287-291. [Crossref]

12. Labrie F, Cusan L, Gomez JL, Côté I, Bérubé R, et al. (2009) Effect of oneweek treatment with vaginal estrogen preparations on serum estrogen levels in postmenopausal women. Menopause 16: 30-36. [Crossref]

13. Santen RJ (2015) Vaginal administration of estradiol: effects of dose, preparation and timing on plasma estradiol levels. Climacteric 18: 1-14.

14. U.S. Food and Drug Administration, Center for Drug Evaluation and Research (2013) department of health and human services, NDA.

15. Osphena (2013) Florham Park. Shionogi Inc, NJ.

16. Senshio (ospemifene) tablets (2014) European Public Assessment Report. European Medicines Agency. London.

17. Bruyniks N, Biglia N, Palacios S, Mueck AO (2017) Systematic indirect comparison of ospemifene versus local estrogens for vulvar and vaginal atrophy. Climacteric 20: 195-204.

18. Portman D, Palacios S, Nappi RE, Mueck AO (2014) Ospemifene, a non-oestrogen selective oestrogen receptor modulator for the treatment of vaginal dryness associated with postmenopausal vulvar and vaginal atrophy: a randomised, placebo-controlled, phase III trial. Maturitas 78: 91-98. [Crossref]

19. Mueck AO, Ruan X, Prasauskas V, Grob P, Ortmann O (2018) a) Treatment of vagina atrophy with estriol and lactobacilli combination: a clinical review. Climacteric 21: 140-147.

20. Simon JA (2011) Identifying and treating sexual dysfunction in postmenopausal women: the role of estrogen. J Womens Health (Larchmt) 20: 1453-1465. [Crossref]

21. Portman DJ, Gass ML (2014) Genitourinary syndrome of menopause: new terminology for vulvovaginal atrophy from the International Society for the Study of Women's Sexual Health and the North American Menopause Society. Menopause 21: 1063-1068.

22. Kingsberg SA, Wysocki S, Magnus L, Krychman ML (2013) Vulvar and vagina atrophy in postmenopausal women: findings from the REVIVE (REal Women's VIews of Treatment Options for Menopausal Vaginal ChangEs) Survey. J Sex Med 10: 17901799

23. Sinha A, Ewies AA (2013) Non-hormonal topical treatment of vulvovaginal atrophy: an up-to-date overview. Climacteric 16: 305-312. [Crossref]

24. Biglia N, Peano E, Sgandurra P (2010) Low-dose vaginal estrogens or vaginal moisturizer in breast cancer survivors with urogenital atrophy: a preliminary study. Gynecol Endocrinology 26: 404-412.

25. Rees M, Perez-Lopez FR, Ceasu I (2012) EMAS clinical guide: Low-dose vaginal estrogens for postmenopausal vaginal atrophy. Maturitas 73: 171-174.

26. Sturdee DW, Panay N, International Menopause Society Writing Group (2010) Recommendations for the management of postmenopausal vaginal atrophy. Climacteric 13: 509-522. [Crossref]

27. Nikander E, Kilkkinen A, Metsa-Heikkila M (2003) A randomized placebo-controlled crossover trial with phytoestrogens in treatment of menopause in breast cancer patients. Obstet Gynecol 101: 1213-1220.

28. Suckling J, Lethaby A, Kennedy R (2006) Local oestrogen for vaginal atrophy in postmenopausal women. Cochrane Database Syst Rev 18: CD001500. 
29. Al-Baghdadi O, Ewies AA (2009) Topical estrogen therapy in the management of postmenopausal vaginal atrophy: an up-to-date overview. Climacteric 12: 91-105.

30. Englund DE, Elamsson KB, Johansson ED (1982) Bioavailability of oestriol. Acta Endocrinol (Copenh) 99: 136-140. [Crossref]

31. Keller PJ, Riedmann R, Fischer M (1980) Oestrone, oestradiol and oestriol content following intravaginal application of oestriol in the postmenopause. Gynakol Rundsch 20: 77-79.

32. Head KA (1998) Estriol: safety and efficacy. Altern Med Rev 3: 101-113. [Crossref]

33. Trinkaus M, Chin S, Wolfman W, Simmons C, Clemons M (2008) Should urogenital atrophy in breast cancer survivors be treated with topical estrogens? Oncologist 13: $222-231$

34. Palacios S, Castelo-Branco C, Cancelo MJ, Vazquez F (2005) Low-dose, vaginally administered estrogens may enhance local benefits of systemic therapy in the treatment of urogenital atrophy in postmenopausal women on hormone therapy. Maturitas 50: 98-104

35. Cicinelli E, de Ziegler D, Bulletti C, Matteo MG, Schonauer LM, et al. (2000) Direct transport of progesterone from vagina to uterus. Obstet Gynecol 95: 403-406. [Crossref]

36. Bachmann G, Lobo RA, Gut R (2008) Efficacy of low-dose estradiol vaginal tablets in the treatment of atrophic vaginitis: a randomized controlled trial. Obstet Gynecol 111: $67-76$

37. Simon J, Nachtigall L, Gut R, Lang E, Archer DF, et al. (2008) Effective treatment of vaginal atrophy with an ultra-low-dose estradiol vaginal tablet. Obstet Gynecol 112: 1053-1060. [Crossref]

38. Ulrich LS, Naessen T, Elia D (2010) VAG-1748 trial investigators. Endometrial safety of ultra-low-dose Vagifem $10 \mathrm{mg}$ in postmenopausal women with vaginal atrophy. Climacteric 13: 228-237

39. Loibl S, Lintermans A, Dieudonné AS, Neven P (2011) Management of menopausal symptoms in breast cancer patients. Maturitas 68: 148-154. [Crossref]

40. Kendall A, Dowsett M, Folkerd E, Smith I (2006) Caution: vaginal estradiol appears to be contraindicated in postmenopausal women on adjuvant aromatase inhibitors. Annals Oncology 17: 584-587.

41. Biglia N, Peano E, Sgandurra P (2010) Low-dose vaginal estrogens or vaginal moisturizer in breast cancer survivors with urogenital atrophy: a preliminary study. Gynecol Endocrinology 26: 404-412

42. Mikkola TS, Tuomikoski P, Lyytinen H, Korhonen P, Hoti F, et al. (2016) Vaginal estradio use and the risk for cardiovascular mortality. Hum Reprod 31: 804-809. [Crossref]

43. Naessen T, Berglund L, Ulmsten U (1997) Bone loss in elderly women prevented by ultralow doses of parenteral 17beta-estradiol. Am J Obstet Gynecol 177: 115-119. [Crossref]

44. Naessen T, Rodriguez-Macias K, Lithell H (2001) Serum lipid profile improved by ultra-low doses of 17 beta-estradiol in elderly women. J Clin Endocrinol Metab 86: 2757-2762. [Crossref]

45. Committee on Practice Bulletins-Gynecology (2012) Management of gynecological issue in women with breast cancer. Obstet Gynecol 119: 666-682.

46. Crandall CJ, Hovey KM, Andrews CA (2018) Breast cancer, endometrial cancer, and cardiovascular events in participants who used vaginal estrogen in the Women's Health Initiative Observational Study. Menopause 25: 11-20.

47. Kuhl H (2005) Pharmacology of estrogens and progestogens: influence of different routes of administration. Climacteric 8: 3-63.

48. van Haaften M, Donker GH, Sie-Go DM (1997) Biochemical and histological effects of vaginal estriol and estradiol applications on the endometrium, myometrium and vagina of postmenopausal women. Gynecol Endocrinology 11: 175-185.

49. Heimer GM, Englund DE (1986) Plasma oestriol following vaginal administration: morning versus evening insertion and influence of food. Maturitas 8: 239-243.

50. van der Vies J (1982) The pharmacology of oestriol. Maturitas 4: 291-299. [Crossref]

51. Ponzone R, Biglia N, Jacomuzzi ME, Maggiorotto F, Mariani L, et al. (2005) Vagina oestrogen therapy after breast cancer: is it safe? Eur J Cancer 41: 2673-2681. [Crossref]

52. Vooijs GP, Geurts TBP (1995) Review of the endometrial safety during intravaginal treatment with estriol. Eur J Obstet Gynecol 62: 101-106.

53. Ortmann O, Doren M, Windler E (2011) Association of Scientific Medical Societies in Germany A. Hormone therapy in perimenopause and postmenopause (HT) Interdisciplinary S3 Guideline, Association of the Scientific Medical Societies in Germany AWMF 015/062-short version. Arch Gynecol Obstet 284: 343-355.
54. Mattsson LA, Cullberg G (1983) Vaginal absorption of two estriol preparations. A comparative study in postmenopausal women. Acta Obstet Gynecol Scand 62: 393-396.

55. Buhling KJ, Eydeler U, Borregaard S (2012) Systemic bioavailability of estriol following single and repeated vaginal administration of $0.03 \mathrm{mg}$ estriol containing pessaries. Drug Research 62: 378-383.

56. Cano A, Estevez J, Usandizaga R (2012) The therapeutic effect of a new ultra low concentration estriol gel formulation $(0.005 \%$ estriol vaginal gel) on symptoms and signs of postmenopausal vaginal atrophy: results from a pivotal phase III study. Menopause 19: 1130-1139.

57. Capobianco G, Wenger JM, Meloni GB (2014) Triple therapy with Lactobacilli acidophili, estriol plus pelvic floor rehabilitation for symptoms of urogenital aging in postmenopausal women. Arch Gynecol Obstet 289: 601-608.

58. Griesser H, Skonietzki S, Fischer T (2012) Low dose estriol pessaries for the treatment of vaginal atrophy: A double-blind placebo-controlled trial investigating the efficacy of pessaries containing $0.2 \mathrm{mg}$ and $0.03 \mathrm{mg}$ estriol. Maturitas $71: 360-368$.

59. Jaisamrarn U, Triratanachat S, Chaikittisilpa S (2013) Ultra-low-dose estriol and lactobacilli in the local treatment of postmenopausal vaginal atrophy: a double-blind randomized trial followed by open-label maintenance therapy. Climacteric 16: 347-355.

60. Caruso S, Cianci S, Amore FF (2016) Quality of life and sexual function of naturally postmenopausal women on an ultralow-concentration estriol vaginal gel. Menopause 23: 47-54.

61. Vardy MD, Lindsay R, Scotti RJ, Mikhail M, Richart RM, et al. (2003) Short-term urogenital effects of raloxifene, tamoxifen, and estrogen. Am J Obstet Gynecol 189: 81-88. [Crossref]

62. Komi J, Lankinen KS, Härkönen P, DeGregorio MW, Voipio S, et al. (2005) Effects of ospemifene and raloxifene on hormonal status, lipids, genital tract, and tolerability in postmenopausal women. Menopause 12: 202-209. [Crossref]

63. Delmanto A, Nahas-Neto J, Nahas EA, de Oliveira ML, Fernandes CE, et al. (2008) Effect of raloxifene on the vaginal epithelium of postmenopausal women. Eur J Obstet Gynecol Reprod Biol 139: 187-192. [Crossref]

64. Polin SA, Ascher SM (2008) The effect of tamoxifen on the genital tract. Cancer Imaging 8: 135-145. [Crossref]

65. Pinkerton JV, Stanczyk FZ (2013) Clinical effects of selective estrogen receptor modulators on vulvar and vaginal atrophy. Menopause 21: 309-319.

66. Kangas L, Unkila M (2013) Tissue selectivity of ospemifene: pharmacologic profile and clinical implications. Steroids 78: 1273-1280.

67. Portman DJ, Bachmann GA, Simon JA (2013) Ospemifene. a novel selective estrogen receptor modulator for treating dyspareunia associated with postmenopausalvulvar and vaginal atrophy. Menopause 20: 623-630.

68. Bachmann GA, Komi JO (2010) Ospemifene effectively treats vulvovaginal atrophy in postmenopausal women: results from a pivotal phase 3 study. Menopause 17: 480-486.

69. Goldstein SR, Bachmann GA, Koninckx PR, Lin VH, Portman DJ, et al. (2014) Ospemifene 12-month safety and efficacy in postmenopausal women with vulvar and vaginal atrophy. Climacteric 17: 173-182. [Crossref]

70. Pinkerton JV, Goldstein SR (2010) Endometrial safety: a key hurdle for selective estrogen receptor modulators in development. Menopause 17: 642-653.

71. Gerbaldo D, Ferraiolo A, Croce S (1991) Endometrial morphology after 12 months of vaginal oestriol therapy in post-menopausal women. Maturitas 13: 269-274.

72. Iosif CS (1992) Effects of protracted administration of estriol on the lower genitourinary tract in postmenopausal women. Arch Gynecol Obstet 251: 115-120.

73. 73) Henriksson L, Stjernquist M, Boquist L, et al. A one-year multicenter study of efficacy and safety of a continuous, low-dose, estradiol-releasing vaginal ring (Estring) in postmenopausal women with symptoms and signs of urogenital aging. Am J Obstet Gynecol 174: 85-92.

74. Naessen T, Rodriguez-Macias K (2002) Endometrial thickness and uterine diameter not affected by ultralow doses of 17 beta-estradiol in elderly women. Am J Obstet Gynecol 186: 944-947.

75. Weisberg E, Ayton R, Darling G, Farrell E, Murkies A, et al. (2005) Endometrial and vaginal effects of low-dose estradiol delivered by vaginal ring or vaginal tablet. Climacteric 8: 83-92. [Crossref]

76. Smith P, Heimer G, Lindskog M, Ulmsten U (1993) Oestradiol-releasing vaginal ring for treatment of postmenopausal urogenital atrophy. Maturitas 16: 145-154.

77. Simon J, Nachtigall L, Ulrich LG, Eugster-Hausmann M, Gut R (2010) Endometrial safety of ultra-low-dose estradiol vaginal tablets. Obstet Gynecol 116: 876-883. [Crossref] 
78. Simon JA, Lin VH, Radovich C, Bachmann GA (2013) Ospemifene Study Group. One-year long-term safety extension study of ospemifene for the treatment of vulvar and vaginal atrophy in postmenopausal women with a uterus. Menopause 20: 418-427.

79. Unlu C, Donders G (2011) Use of lactobacilli and estriol combination in the treatment of disturbed vaginal ecosystem: a review. J Turk Ger Gynecol Assoc 12: 239-246.

80. Shen J, Song N, Williams CJ (2016) Effects of low dose estrogen therapy on the vaginal microbiomes of women with atrophic vaginitis. Sci Rep 6: 243-280.

81. Witkin SS, Linhares IM, Giraldo P (2007) Bacterial flora of the female genital tract: function and immune regulation. Best Pract Res Clin Obstet Gynaecol 21: 347-354.

82. Reid G, Younes JA, van der Mei HC (2011) Microbiota restoration: natural and supplemented recovery of human microbial communities. Nat Rev Microbiol 9: 27-38.

83. Kaiser RR, Michael-Hepp J, Weber W (2000) Absorption of estriol from vaginal tablets after single and repeated application in healthy, postmenopausal women. Therapiewoche 3: 2-8.
84. Donders G, Neven P, Moegele M (2014) Ultra-low-dose estriol and Lactobacillus acidophilus vaginal tablets (Gynoflor) for vaginal atrophy in postmenopausal breast cancer patients on aromatase inhibitors: pharmacokinetic, safety, and efficacy phase I clinical study. Breast Cancer Res Treat 145: 371-379.

85. Furuhjelm M, Karlgren E, Carlström K (1980) Intravaginal administration of conjugated estrogens in premenopausal and postmenopausal women. Int J Gynaecol Obstet 17: 335-339

86. Samsioe G (1998) Urogenital aging--a hidden problem. Am J Obstet Gynecol 178: S245-249. [Crossref]

87. Sousa MS, Peate M, Jarvis S (2017) A clinical guide to the management of genitourinary symptoms in breast cancer survivors on endocrine therapy. Ther Adv Med Oncol 9: 269-285.

Copyright: C2018 Ruan X. This is an open-access article distributed under the terms of the Creative Commons Attribution License, which permits unrestricted use, distribution, and reproduction in any medium, provided the original author and source are credited. 